\title{
PRIMARY ANGLE-CLOSURE GLAUCOMA WITH CAPSULAR EXFOLIATION OF THE LENS*
}

\author{
BY \\ RONALD F. LOWE \\ Ophthalmic Research Institute of Australia, and Royal Victorian Eye and Ear Hospital, Melbourne, Australia
}

CAPSUlaR exfoliation (or pseudo-exfoliation) of the lens is a readily recognized condition that has amassed a formidable literature. Its association with chronic glaucoma has caused much interest and controversy. Sugar (1957) provided an extensive review. However, the relationship between capsular exfoliation of the lens and primary angle-closure glaucoma has received little attention.

\section{Investigations}

From the Glaucoma Unit of The Royal Victorian Eye and Ear Hospital, Melbourne, Australia, 250 patients with primary angle-closure glaucoma were examined by the writer. Their symptomatology and the clinical types of glaucoma have previously been described (Lowe, 1962). The Table shows the type of primary angle-closure glaucoma diagnosed when the patients presented.

TABLE

TYPE OF GLAUCOMA IN 250 CASES OF PRIMARY ANGLE-CLOSURE GLAUCOMA WHEN PATIENT FIRST PRESENTED

\begin{tabular}{l|c}
\hline \multicolumn{1}{c|}{ Type of Glaucoma } & No. of Cases \\
\hline Subacute episodes & 16 \\
Creeping angle-closure & 17 \\
Acute angle-closure & 215 \\
Acute angle-closure combined with chronic simple glaucoma & 2 \\
\hline \multicolumn{1}{c|}{ Total } & 250 \\
\hline
\end{tabular}

All examinations included slit-lamp biomicroscopy and gonioscopy, during which signs of capsular exfoliation were sought. From the 250 patients examined only seven were found with capsular exfoliation. It is possible that a few may have been overlooked because all pupils could not be dilated fully but, as operations were routinely performed upon both eyes and pupil dilatation was part of the post-operative treatment, it is unlikely that many escaped detection.

\section{Nationality}

As Australia has received many immigrants from numerous countries the nationality of the patients was recorded. Of the seven with capsular exfoliation, six were Australianborn of United Kingdom stock and one was a Latvian immigrant. This is of some interest because capsular exfoliation is said to be very uncommon in England (Thomassen, 1949; Evans, 1958), but Garrow (1938) found eight cases (16 per cent.) in 51 patients with chronic glaucoma in Scotland. 
Sex

The seven patients comprised five females and two males. This proportion concurs with that found for angle-closure glaucoma in the community (Lowe, 1962) and not with that for chronic simple glaucoma. Further, in an ophthalmic clinic from the same hospital and from 88 patients with chronic simple glaucoma, Gillies (1963) found nineteen with capsular exfoliation-ten females and nine males.

\section{Age at Diagnosis}

The seven patients with capsular exfoliation had an age range of 58 to 88 years (mean 73) The other 243 patients with primary angle-closure glaucoma had an age range of 22 to 88 years (mean 61). Thus capsular exfoliation occurs only in a few of the oldest patients with primary angle-closure glaucoma.

\section{Clinical Types}

Of the total of seven patients, four females had bilateral capsular exfoliation when they presented with unilateral acute angle-closure glaucoma.

Of the three with unilateral capsular exfoliation, one man presented after two episodes of subacute angle-closure in the eye without capsular exfoliation. This eye showed no evidence of chronic simple glaucoma for the 2 years which elapsed before he died aged 79 years, but his other eye, which had capsular exfoliation, had severe chronic simple glaucoma for 8 years, during which thrombosis of the central retinal artery and absolute glaucoma supervened. The other man presented with acute angle-closure glaucoma in the eye with capsular exfoliation. The third was a female patient with bilateral acute angle-closure glaucoma, but with capsular exfoliation in the left eye only.

\section{Incidence of Chronic Simple Glaucoma}

Of the 250 patients with primary angle-closure glaucoma, two had been receiving treatment for chronic simple glaucoma before the onset of an acute attack. Another five were found to have additional chronic simple glaucoma after attacks of acute angle-closure had been relieved. None of these seven patients with mixed glaucoma had capsular exfoliation.

Of the seven patients presenting with some form of primary angle-closure glaucoma and capsular exfoliation, one had advanced chronic simple glaucoma and capsular exfoliation in the other eye and one had bilateral capsular exfoliation with normal tensions and normal fields but $C$ values of $0 \cdot 12$ in each eye and $P o / C$ values of 141 and 106 . The remaining five showed no suggestion of chronic simple glaucoma when this was especially sought (and two of these patients have now died, each aged 89 years).

From an ophthalmic clinic in the same hospital, Gillies (1963) reported nineteen cases of capsular exfoliation among 88 patients who presented with chronic simple glaucoma.

\section{Conclusions}

Despite all that has been written about capsular exfoliation (or pseudo-capsular exfoliation) of the lens, the condition remains an enigma. Its incidence appears to vary considerably among different races, especially when considered in cases of chronic glaucoma having open anterior chamber angles. In this connexion it is said to be very uncommon in England but it is not uncommon in Australia among those whose close forebears came from the United Kingdom. Although it has been found in approximately 20 per cent. of a series of 88 patients with chronic simple glaucoma attending The Royal Victorian Eye and Ear Hospital in Melbourne, it has been 
diagnosed in less than 3 per cent. of 250 patients with primary angle-closure glaucoma who have attended the same hospital. Its sex incidence and age incidence also indicate that it has no significant concomitant relationship with primary angleclosure glaucoma.

\section{Summary}

From 250 cases of primary angle-closure glaucoma seven were found to have capsular exfoliation of the lens-three unilateral, four bilateral. In Victoria, Australia, it appears to have no concomitant relationship with primary angle-closure glaucoma and when it does occur it is found in only a few of the elderly members of this disease group.

These investigations were part of Research Project No. 14 of the Ophthalmic Research Institute of Australia, and Research Project No. 13 of The Royal Victorian Eye and Ear Hospital.

\section{REFERENCES}

Evans, P. JAMEson (1958). Trans. ophthal. Soc. Aust. 1957, 17, 27.

GARROW, A. (1938). Brit. J. Ophthal., 22, 214.

GILLIES, W. E. (1963). Trans. ophthal. Soc. Aust. 1962, 22, 120.

LOWE, R. F. (1962). Ibid. 1961, 21, 65.

SugAR, H. S. (1957). "The Glaucomas", 2nd ed. Hoeber-Harper, New York.

ThOMASSEN, T. L. (1949). Acta ophthal. (Kbh), 27, 423. 